\title{
Formamide-Free Genomic in situ Hybridization Allows Unambiguous Discrimination of Highly Similar Parental Genomes in Diploid Hybrids and Allopolyploids
}

\author{
Tae-Soo Jang Hanna Weiss-Schneeweiss \\ Department of Botany and Biodiversity Research, University of Vienna, Vienna, Austria
}

\section{Key Words}

Allopolyploid · Formamide-free GISH · Hybrid ·

Melampodium · Prospero · Standard GISH protocol

\begin{abstract}
Polyploidy and hybridization play an important role in plant diversification and speciation. The application of genomic in situ hybridization (GISH) allows the identification of parental genomes in hybrids, thus elucidating their origins and allowing for analysis of their genomic evolution. The performance of GISH depends on the similarity of the parental genomes and on the age of hybrids. Here, we present the formamidefree GISH (ff-GISH) protocol applied to diploid and polyploid hybrids of monocots (Prospero, Hyacinthaceae) and dicots (Melampodium, Asteraceae) differing in similarity of the parental genomes and in chromosome and genome sizes. The efficiency of the new protocol is compared to the standard GISH protocol. As a result, ff-GISH allowed efficient labeling and discrimination of the parental chromosome sets in diploid and allopolyploid hybrids in Prospero autumnale species complex. In contrast, the standard GISH protocol failed to differentiate the parental genomes due to high levels of similar repetitive DNA. Likewise, an unambiguous identification
\end{abstract}

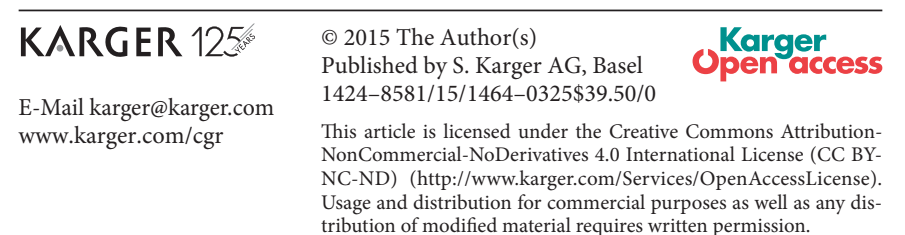

of parental genomes in allotetraploid Melampodium nayaritense (Asteraceae) was possible after $\mathrm{ff}-\mathrm{GISH}$, whereas the standard GISH hybridization performance was suboptimal. The modified method is simple and non-toxic and allows the discrimination of very similar parental genomes in hybrids. This method lends itself to modifications and improvements and can also be used for FISH.

\section{(C) 2015 The Author(s)}

Published by S. Karger AG, Basel

Polyploidy and hybridization play an important role in plant diversification and speciation [Soltis and Soltis, 2009]. The major challenge in studying hybrids at both the diploid and polyploid level is the unambiguous identification of parental taxa, which subsequently contributes to in-depth analyses of the evolution of polyploid genomes. Various methods are employed in this context, including morphological analyses, phylogenetic analyses of nuclear and plastid DNA markers, fingerprinting methods, and cytogenetics [Lihová et al., 2007; WeissSchneeweiss et al., 2012]. The latter encompasses classical and molecular methods, most notably fluorescence in situ hybridization (FISH) and genomic in situ hybridization (GISH). In particular, GISH allows differential labeling of 


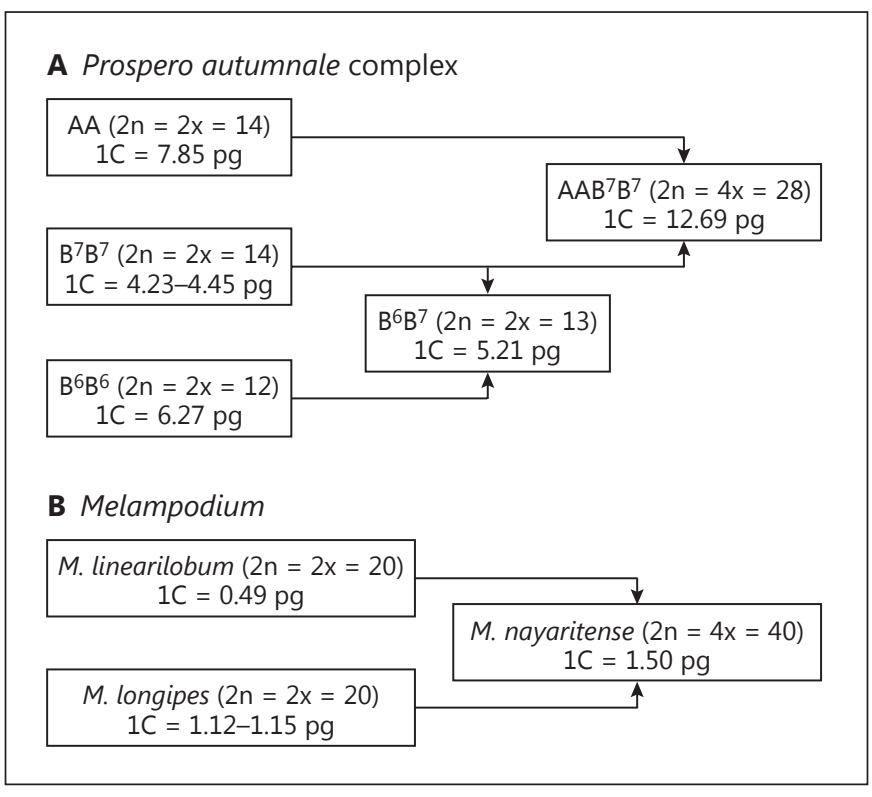

Fig. 1. Schematic showing the representation of genome evolution in the analyzed taxa of plant genera Prospero (A) and Melampo$\operatorname{dium}(\mathbf{B})$, respectively.

the parental genomes in hybrids. The success rate of GISH, however, strongly depends on the similarity of the parental genomes (the more similar, the more difficult it becomes to distinguish the parental genomes) and on the evolutionary age of the hybrids (the older the hybrids, the more difficult it is to distinguish the parental genomes) [Lim et al., 2005]. Since its first application [Schwarzacher et al., 1989], various GISH protocols have been developed to successfully differentiate parental genomes in plants [Choi et al., 2008; Pendinen et al., 2008; Mandáková et al., 2014]. However, most of these are optimized specifically for certain plant groups [Renny-Byfield et al., 2010; Jeridi et al., 2011; Chester et al., 2012, 2015]. The major limitation of the use of GISH in plant hybrids is poor performance when closely related parental genomes (species or varieties) which share the majority of the repetitive DNA elements are involved in hybridization.

Diploid and polyploid hybrids of 2 plant groups, representing monocots and dicots, of known parental origin were analyzed in the current study (fig. 1) [Weiss-Schneeweiss et al., 2012; Jang et al., 2013]. Specifically, diploid homoploid and polyploid hybrids of the monocot Prospero autumnale complex (Hyacinthaceae) with relatively large genome sizes, and polyploid hybrids of the dicotyledonous genus Melampodium (Asteraceae) with small ge- nome sizes were targeted. The circum-Mediterranean $P$. autumnale complex, 1 of the 3 species in the genus, is cytologically variable [Jang et al., 2013]. The 4 diploid cytotypes of this complex differ in the combination of their basic chromosome number and genome size [Jang et al., 2013]. Cytotypes AA and $B^{7} B^{7}$ [Vaughan et al., 1997; Jang et al., 2013] possess a basic chromosome number of $x=7$ but differ in genome size, with cytotype AA having a 70\% larger genome than $\mathrm{B}^{7} \mathrm{~B}^{7}$ (1C of $7.85 \mathrm{pg}$ and $4.23 / 4.45 \mathrm{pg}$, respectively) [Jang et al., 2013]. Cytotypes $\mathrm{B}^{6} \mathrm{~B}^{6}$, endemic to Crete, and $\mathrm{B}^{5} \mathrm{~B}^{5}$, endemic to Libya, underwent independent reductions of base chromosome numbers to $\mathrm{x}=6$ and $\mathrm{x}=5$, respectively, as well as increases of genome sizes (1C of $6.27 \mathrm{pg}$ and $4.68 \mathrm{pg}$, respectively) [Jang et al., 2013]. Analyses of the repetitive DNA fraction of all 4 diploid cytotypes revealed the presence of one widespread, but variable in copy number, satellite DNA PaB6 [Emadzade et al., 2014], and similar relative proportions of all major lineages of transposable elements [Weiss-Schneeweiss et al., unpubl. data]. Although the 4 cytotypes form phylogenetically distinct groups [Jang et al., 2013], they are nearly morphologically indistinguishable. Almost all diploid cytotypes, except for the $\mathrm{B}^{5}$ genome, form polyploids, allopolyploids (combinations of $\mathrm{A}$ and $\mathrm{B}^{7}$ as well as $\mathrm{B}^{6}$ and $\mathrm{B}^{7}$ genomes), and autopolyploids originating from the $\mathrm{B}^{7}$ genome [Vaughan et al., 1997]. In addition, all diploid cytotypes can be crossed and produce viable diploid homoploid hybrids [Taylor, 1997; Jang et al., 2013].

The dicotyledonous genus Melampodium (Asteraceae), endemic to Mexico and adjacent territories, encompasses 40 species in 8 sections [Stuessy et al., 2011]. Five different basic chromosome numbers occur in diploids of this genus, $\mathrm{x}=9,10,11,12$, and 14 [Weiss-Schneeweiss et al., 2009]. The largest section, Melampodium, comprises 20 species grouped into 5 series, all with a base chromosome number of $x=10$. One of the series of this section, series Sericea, contains exclusively tetraploid and hexaploid species, all of hybrid origin. The parental origins of these allopolyploids were inferred using phylogenetic analyses of nrITS, 5S rDNA spacer (NTS), plastid regions, and the low copy gene $P g i C$, and general trends in rDNA evolution were inferred via FISH [Blöch et al., 2009; Weiss-Schneeweiss et al., 2009, 2012; Stuessy et al., 2011]. The diploid species M. linearilobum and M. longipes (although its sister taxon $M$. americanum could not be excluded) were identified as the parental species of the allotetraploid $M$. nayaritense [Weiss-Schneeweiss et al., 2012]. The 2 diploids share the same base chromosome number of $\mathrm{x}=10$, but differ in genome size ( $1 \mathrm{C}$ of $0.49 \mathrm{pg}$ vs. $1.12 \mathrm{pg}$, respectively) [Weiss-Schneeweiss et al., 2012] 
and consequently in the proportion and the composition of the repetitive DNA sequences [McCann et al., unpubl. data].

The aim of this study was to develop a simple GISH protocol that could be used across various plant groups and would effectively distinguish parental genomes in hybrids regardless of their parental genome similarity, genome size, repeat composition, and taxonomic affiliations. Most available protocols include organic solvents, most commonly formamide, in hybridization mixes which lowers the effective DNA melting temperature and allows hybridization at lower temperatures without compromising the stringency [Meinkoth and Wahl, 1984]. Formamide, however, prolongs the time of hybridization by slowing down the rate of renaturation [Berndt et al., 1996; Blake and Delcourt, 1996]. The performance of newly established formamide-free GISH protocol (from now on called ff-GISH) in the 2 above-mentioned plant groups was compared to the commonly used formamidebased GISH protocol [Schwarzacher and Heslop-Harrison, 2000].

\section{Materials and Methods}

\section{Plant Material}

P. autumnale Complex. A diploid homoploid hybrid of the genomic composition $\mathrm{B}^{6} \mathrm{~B}^{7}$ ( $\mathrm{F}_{1}$ cross between diploid cytotypes $\mathrm{B}^{6} \mathrm{~B}^{6}$ and $\mathrm{B}^{7} \mathrm{~B}^{7} ; \mathrm{H} 364$, Crete, Greece) and an allopolyploid hybrid $\mathrm{AAB}^{7} \mathrm{~B}^{7}$ (H603, Algarve, Spain) were used for analyses (fig. 1A). Plants were cultivated in the Botanical Garden of the University of Vienna (HBV).

Melampodium. Allotetraploid M. nayaritense (M115, Nayarit, Mexico) was analyzed (fig. 1B). Seeds were collected from natural populations in Mexico in August 2013 and germinated, and plantlets were cultivated in the Botanical Garden of the University of Vienna (HBV).

Actively growing root meristems of all plants were harvested, pretreated with colchicine (for Prospero) [Jang et al., 2013] or 8-hydroxyquinoline (for Melampodium) [Weiss-Schneeweiss et al., 2009], fixed in 3:1 ethanol:acetic acid mixture, and stored at $-20^{\circ} \mathrm{C}$ until use.

\section{Genomic in situ Hybridization}

Enzymatic digestion of fixed root meristems and chromosome preparations were made as described earlier [Weiss-Schneeweiss et al., 2012; Jang et al., 2013]. GISH was carried out following standard chromosome pretreatment with RNAse and pepsin [WeissSchneeweiss et al., 2012].

DNAs of putative parental genomes were used as probes (fig. 1). Total genomic DNAs from P. autumnale diploid cytotypes AA, $\mathrm{B}^{6} \mathrm{~B}^{6}$, and $\mathrm{B}^{7} \mathrm{~B}^{7}$ as well as $M$. linearilobum $(2 \mathrm{x})$ and $M$. longipes $(2 \mathrm{x})$ were isolated using the CTAB method [Doyle and Doyle, 1987; Jang et al., 2013] and sheared at $98^{\circ} \mathrm{C}$ for $5 \mathrm{~min}$. Approximately $1 \mu \mathrm{g}$ of genomic DNA of each cytotype was labeled with either di- goxigenin or biotin using a nick translation kit (Roche, Vienna, Austria). The fragment length of the resulting probes was about $100 \mathrm{bp}$. Probes were purified via ethanol precipitation and resuspended in water.

The GISH mix consisted of $10 \%$ dextran sulfate (Sigma Aldrich, Vienna, Austria), $0.02 \times$ SSC, $1 \%$ salmon sperm DNA, and $3-4 \mathrm{ng} / \mu \mathrm{l}$ of each of 2 labeled genomic DNA probes. The hybridization mix was denatured at $98^{\circ} \mathrm{C}$ for $5 \mathrm{~min}$ and stored at $4{ }^{\circ} \mathrm{C}$. Hybridization mix ( $10 \mu \mathrm{l}$ per slide containing $30-40 \mathrm{ng}$ of each of the labeled genomic probes) was applied to each slide and sealed under a coverslip. Combined denaturation of the material on the slide and hybridization mix was performed at $72^{\circ} \mathrm{C}$ for $4 \mathrm{~min}$, and they were allowed gradually to cool down to $37^{\circ} \mathrm{C}\left(1 \mathrm{~min}\right.$ at $65^{\circ} \mathrm{C}$, $1 \mathrm{~min}$ at $55^{\circ} \mathrm{C}$, and $1 \mathrm{~min}$ at $45^{\circ} \mathrm{C}$ ) on a PCR in situ block. Hybridization was carried out for $12-24 \mathrm{~h}$ at $37^{\circ} \mathrm{C}$. Stringent washes were performed 3 times in $2 \times \mathrm{SSC}$ at $42^{\circ} \mathrm{C}$ for $3 \mathrm{~min}$ each. Slides were incubated for $20 \mathrm{~min}$ at $37^{\circ} \mathrm{C}$ in blocking solution containing $5 \%$ bovine serum albumin (BSA) and Tween 20 in $2 \times$ SSC buffer. Probes labeled with digoxigenin were detected using $1 \mathrm{ng} / \mu \mathrm{l}$ FITCconjugated antidigoxigenin (Roche), and biotin was detected using $3 \mathrm{ng} / \mu \mathrm{l} \mathrm{Cy} 3$-conjugated ExtraAvidin (Sigma), both in $2 \times \mathrm{SSC}$ containing $5 \%(\mathrm{w} / \mathrm{v}) \mathrm{BSA}$ and $0.5 \%$ Tween 20 for $1 \mathrm{~h}$ at $37^{\circ} \mathrm{C}$. Subsequently, preparations were washed twice in $2 \times \mathrm{SSC}$ and once in $2 \times$ SSC, $0.5 \%$ Tween 20 at $42^{\circ} \mathrm{C}, 3$ min each. DNA was counterstained with $2 \mathrm{ng} / \mu \mathrm{l}$ DAPI (4',6-diamidino-2-phenylindole) and mounted in Vectashield antifade medium (Vector Laboratories, Burlingame, Calif., USA). Preparations were analyzed with an AxioImager M2 epifluorescent microscope (Carl Zeiss, Vienna, Austria), and images were captured with a CCD camera and processed using AxioVision ver. 4.8 (Carl Zeiss) using only those functions that apply equally to the entire image. A detailed step-by-step protocol is available as online supplementary material (online suppl. file 1; see www.karger.com/doi/10.1159/000441210 for all online suppl. material).

As a control, GISH using the standard hybridization mix [Schwarzacher and Heslop-Harrison, 2000] was performed in parallel. To test the effect of the formamide-free hybridization solution on the denaturation of the chromosomes, additional experiments were performed where denaturation of the preparations was performed in Coplin jars in 70\% formamide solution and only then was the denatured hybridization mix added to the slides. This experiment was again performed for both standard and formamidefree hybridization mixes in parallel.

The performance of the present method has also been compared to another formamide-free GISH protocol applied earlier to Tragopogon chromosomes (Asteraceae) [Chester et al., 2012, 2015]. The hybridization mix composition and hybridization conditions from Chester et al. [2012] were used in this experiment whereas chromosome preparation, DNA labeling, and detection for practical and comparative reasons followed the ff-GISH protocol.

The $P$. autumnale hybrid $\mathrm{B}^{6} \mathrm{~B}^{7}$ contained significant amounts of the tandem repeat $\mathrm{PaB}$, nearly all contributed by the $\mathrm{B}^{6}$ genome [Emadzade et al., 2014]. In order to reduce the effects of the strong hybridization of $\mathrm{PaB} 6$ to pericentric chromosomal regions, which negatively affected image acquisition (signal-to-noise ratio), unlabeled monomers of the satellite DNA PaB6 were added to the hybridization mix as blocking DNA in a concentration $20 \times$ higher than that of the labeled probes ( $600-800 \mathrm{ng}$ per slide). Again, this experiment was performed for both the standard mix and formamide-free hybridization mix. 
Fig. 2. Comparison of standard formamidecontaining $(\mathbf{B}, \mathbf{E}, \mathbf{H})$ and formamide-free $(\mathbf{A}, \mathbf{C}, \mathbf{D}, \mathbf{F}, \mathbf{G}, \mathbf{I}) \mathrm{GISH}$ protocols in the plant genera Melampodium (Asteraceae) and Prospero (Hyacinthaceae). A-C Diploid homoploid hybrid $\mathrm{B}^{6} \mathrm{~B}^{7}$ of $P$. autumnale complex. D-F Allotetraploid $\mathrm{AAB}^{7} \mathrm{~B}^{7}$ of $P$. autumnale complex. G-I Allotetraploid $M$. nayaritense. A, D, G Denaturation and hybridization in formamide-free hybridization mix (ff-GISH ${ }^{1}$ ). C, F, I Denaturation in $70 \%$ formamide and hybridization in formamide-free hybridization mix (ff-GISH ${ }^{2}$ ). Images $\mathbf{A}$ and B-1 show result of hybridization using pericentric satellite $\mathrm{PaB} 6$ as unlabeled blocking DNA. Image B-2 shows hybridization without blocking satellite PaB6. Scale bars $=5 \mu \mathrm{m}$.
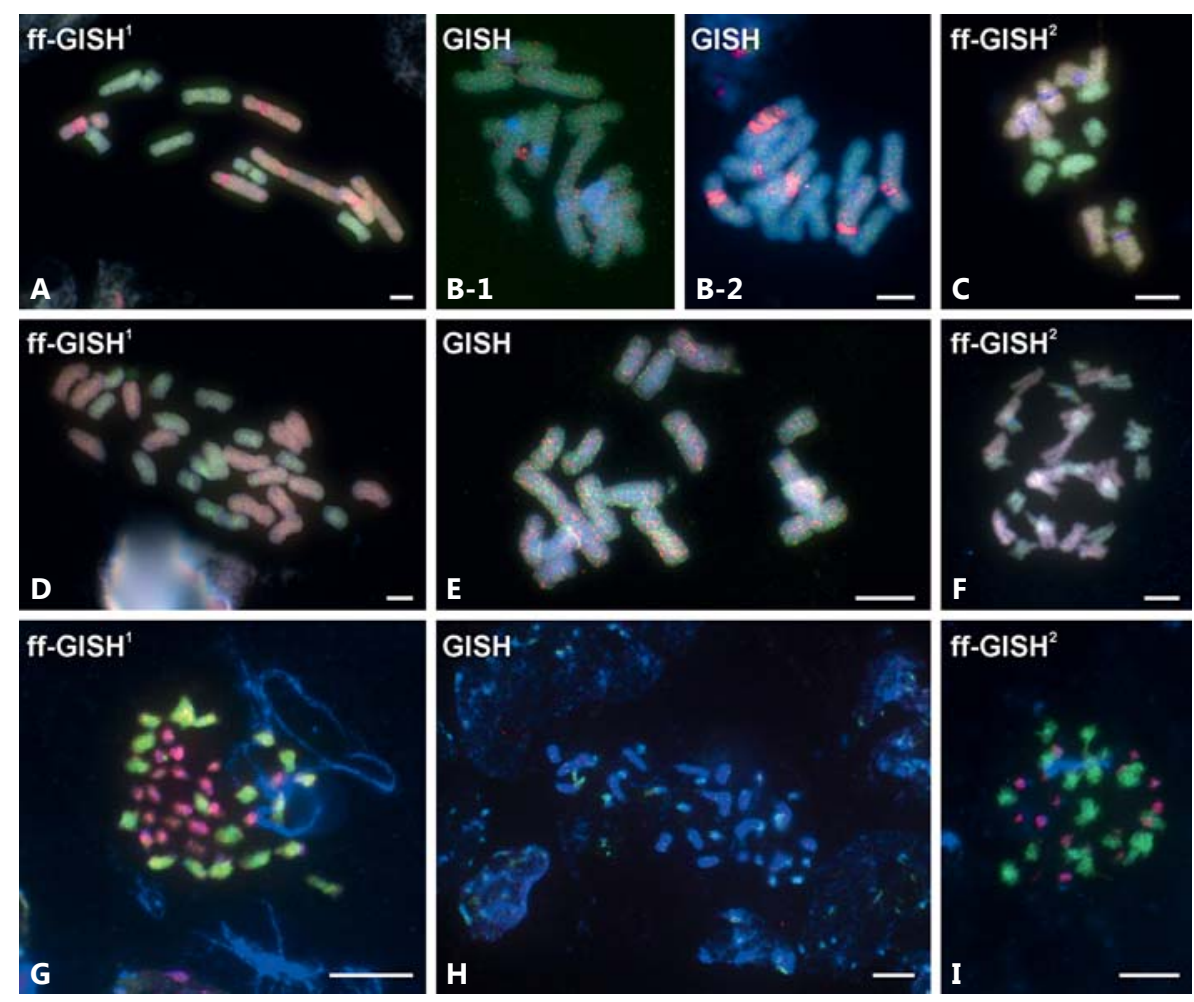

\section{Results and Discussion}

\section{P. autumnale Complex}

In $P$. autumnale, a diploid homoploid hybrid of $\mathrm{B}^{6} \mathrm{~B}^{7}$ origin (fig. $2 \mathrm{~A}-\mathrm{C}$ ) as well as an allotetraploid $\mathrm{AAB}^{7} \mathrm{~B}^{7}$ (fig. 2D, F) were analyzed (fig. 1A). Only some of the individual chromosomes of the parental cytotypes could be identified by their distinct morphology or size, whereas a subset of chromosomes always remained unassigned in both hybrids. The standard GISH method did not allow efficient hybridization and discrimination of the parental chromosome sets, neither in the allopolyploid $\mathrm{AAB}^{7} \mathrm{~B}^{7}$ (fig. 2E) nor in the diploid hybrid $\mathrm{B}^{6} \mathrm{~B}^{7}$ (fig. 2B). This result was expected due to the high degree of similarity of repetitive DNA fractions of the individual cytotypes (over 90\%) [Weiss-Schneeweiss et al., unpubl. data] despite quite significant genome size variation [Jang et al., 2013]. The new ff-GISH protocol, however, allowed unambiguous differentiation of the 2 parental chromosome sets (fig. 2A, D; online suppl. figs. 1,2) both in the diploid and allotetraploid hybrid.

Satellite $\mathrm{PaB6}$ is highly amplified in the $\mathrm{B}^{6}$ genome but is nearly absent from the $\mathrm{B}^{7}$ genome [Emadzade et al., 2014], and its strong hybridization to pericentric regions of chromosomes of $\mathrm{B}^{6}$ origin in $\mathrm{B}^{6} \mathrm{~B}^{7}$ hybrid genome hin- ders acquisition of well contrasted images (fig. 2B-2). When GISH was performed only with 2 labeled genomic parental DNAs, satellite DNA signals after GISH were much stronger than any other signals. Addition of an excess of unlabeled, PCR-amplified monomers of $\mathrm{PaB} 6$ to the hybridization mix reduced the intensity of most $\mathrm{PaB} 6$ signals and allowed the acquisition of good quality GISH images (fig. 2A, B-1, C). The only prerequisite for such a procedure is the identification and isolation of most abundant satellite DNAs from the genomes of interest. It is not absolutely necessary for the success of ff-GISH procedure, but it certainly results in more optimal performance of the method.

\section{Melampodium}

The allotetraploid $M$. nayaritense $(2 \mathrm{n}=4 \mathrm{x}=40)$ originated from a cross between diploid $M$. longipes (or less likely its sister species $M$. americanum) and diploid $M$. linearilobum (fig. 1B). Its karyotype is bimodal with 20 very small chromosomes contributed by $M$. linearilobum and 20 medium-sized chromosomes of $M$. longipes (fig. 2G-I). Due to this size difference, the parental chromosomes can easily be identified in the hybrid even in unstained preparations using phase contrast imaging, helping to verify the GISH performance. 
GISH in allotetraploid M. nayaritense $(2 \mathrm{n}=4 \mathrm{x}=40$; fig. 2G-I) using the standard hybridization mix containing formamide (standard hybmix) [Schwarzacher and Heslop-Harrison, 2000] resulted in uneven chromosome labeling with low signal-to-noise ratio (fig. $2 \mathrm{H}$ ). The small chromosomes of $M$. linearilobum origin were preferentially stronger labeled. Detailed analyses concerning genome evolution were hardly possible under these conditions. In contrast, the new ff-GISH method allowed a very clear and unambiguous labeling of the 2 parental genomes in $M$. nayaritense (fig. 2G; online suppl. figs. 1,2).

\section{Formamide-Free GISH}

The effective melting temperature of DNA (Eff $\mathrm{T}_{\mathrm{m}}$ ) is controlled by the sodium concentration and is close to $100^{\circ} \mathrm{C}$ in water. Consequently, the corresponding hybridization temperature would be at $60-70^{\circ} \mathrm{C}$ which, over a prolonged period of time, causes deterioration of chromosome and nuclei morphology. Therefore, organic solvents, most commonly formamide, have been used in hybridization mixes to lower the effective DNA melting temperature by reducing the thermal stability of doublestranded polynucleotides. Hybridization in the presence of formamide can be performed at lower temperatures without compromising the stringency ( $1 \%$ of formamide reduces the melting temperature of nucleic acid duplexes by $0.72^{\circ} \mathrm{C}$ ) [Meinkoth and Wahl, 1984]. Typically, hybridization mixes contain $50 \%$ formamide which allows hybridization at $37-42^{\circ} \mathrm{C}$ with relatively high stringency. Formamide, however, also slows down the rate of renaturation and thus prolongs the time of hybridization considerably [Berndt et al., 1996; Blake and Delcourt, 1996].

Formamide in high concentration with moderate heat denaturation has been established as the standard in situ hybridization protocol ('formamide protocol'). This treatment allows for high stringency, i.e. minor binding sites of DNA probes can be suppressed so that only specific binding site(s) remain labeled. However, formamidefree hybridization mixes have been successfully applied for hybridization of single-stranded oligonucleotide probes (so called Fast FISH) [Berndt et al., 1996; Durm et al., 1996]. It has been argued that omission of formamide enhanced the sensitivity of probe detection. Recently, a formamide-free ISH mix has been developed for gene aberration tests in diagnostics, but formamide has been substituted with other non-toxic organic solvents (e.g. ethylene carbonate) [Matthiesen and Hansen, 2012].

In the absence of formamide, hybridization time and temperature are the most sensitive parameters for the hybridization process. Previously it was demonstrated that

ff-GISH in Diploid Hybrids and Allopolyploids both these parameters quantitatively influence the hybridization behavior of $a$-satellite probes and how this effect can be used to discriminate major and minor binding sites [Durm et al., 1996]. However, additional factors, such as the degree of condensation and 'aging' of the chromosomal targets, the composition and the $\mathrm{pH}$ of the buffer, and the type of chemical modifications used to label the DNA probes were also suggested to play a role in optimizing the hybridization efficiency. Lower hybridization temperatures $\left(40\right.$ vs. $70^{\circ} \mathrm{C}$ for human $\alpha$-satellite DNA in the non-formamide mix) and prolonged hybridization times ( $2 \mathrm{~h}$ vs. $15 \mathrm{~min}$ ) resulted in hybridization of nearly all $\alpha$-satellite DNA loci (low stringency) [Durm et al., 1996].

In comparison to the standard formamide-GISH hybmix, our ff-GISH procedure resulted not only in a much better discrimination of parental genomes in GISH experiments but also in stronger signals in FISH experiments with non-genomic probes like satellite DNA $\mathrm{PaB6}$ or $35 \mathrm{~S}$ and $5 \mathrm{~S}$ rDNA probes (both in independent FISH experiments and after reprobing of the GISH preparations; data not shown). To test if this improvement is (partially) due to the changed denaturation conditions in the absence of formamide, we denatured the preparations in a Coplin jar in $70 \%$ formamide prior to hybridization with either standard or ff-GISH mix, leaving all other steps and conditions of ff-GISH unchanged. No obvious differences were noted after the denaturation in formamide, except for a slightly more distorted chromosome structure and morphology (fig. 2C, F, I). Thus, the better performance of the ff-GISH is the result of the hybridization itself rather than the denaturation. The use of the formamide-free hybridization mix for FISH should, however, be further optimized by increasing the temperature and decreasing the time of hybridization to obtain an optimal signal-to-noise ratio of the specific probe to be hybridized. The less target-specific the probe (e.g. specific retroelement family), the more stringent conditions should be applied.

The melting temperature of nucleic acid hybrids depends not only on formamide but also on the concentration of monovalent cations (especially $\mathrm{Na}^{+}$) and the GC content of the hybridized region, among others [Meinkoth and Wahl, 1984; Blake and Delcourt, 1996]. The increased sensitivity of the formamide-free in situ hybridization presented here mostly relies on the higher Eff $\mathrm{T}_{\mathrm{m}}$ of DNA hybrids, but also on the very low final concentration of SSC $(0.02 \times)$. Low $\mathrm{Na}^{+}$concentration allows for faster renaturation of the DNA, while the absence of formamide increases the renaturation temperature. Calculations of 
Eff $\mathrm{T}_{\mathrm{m}}$ for both standard and new formamide-free hybridization mixes yielded very similar values: (a) 53.9 and $56.7^{\circ} \mathrm{C}$, respectively, for $40 \%$ GC content of the hybridized regions, and (b) 62.1 and $64.9^{\circ} \mathrm{C}$, respectively for $60 \%$ GC content. The homology percentages (stringency of hybridization) at a hybridization temperature of $37^{\circ} \mathrm{C}$ corresponded to 87.9 and $85.9 \%$ (for $40 \%$ GC) and 82 and $80 \%$ (for $60 \% \mathrm{GC}$ ) for standard and formamide-free hybridization mixes, respectively. Since we do not have detailed information about GC content of the analyzed genomes, it is difficult to address this aspect. However, all of these Eff $\mathrm{T}_{\mathrm{m}}$ allow sufficient and efficient denaturation at a standard $72^{\circ} \mathrm{C}$ temperature.

Due to the low salt concentration and the absence of formamide, the hybridization efficiency is higher and the rate faster, which makes the whole procedure more efficient. Additionally, the low hybridization temperature and the prolonged hybridization time (overnight, mostly for convenience reasons in order not to extend the experiment over $10 \mathrm{~h}$ per working day) might allow maximal saturation of genomic hybridization. Different times of hybridization (10-24 h) have been tested, and they did not significantly change the efficiency of the GISH. It is possible that the time of hybridization could still be shortened.

In comparison, the GISH protocol applied to Tragopogon [Chester et al., 2012] and based on the formamidefree FISH protocol developed for maize [Kato et al., 2004; Birchler et al., 2008] also allowed labeling of the parental genomes both in Prospero and in Melampodium (online suppl. fig. 3). The differentiation of the 2 genomes was comparable to their differentiation after ff-GISH in P. autumnale, although slightly less pronounced. In $M$. nayaritense, however, although the parental genomes were differentially labeled, chromosome morphology after GISH was suboptimal due to significant DNA loss (as judged by very poor DAPI labeling). The 2 protocols, the newly presented ff-GISH protocol and protocol of Chester et al. [2012], differ in several steps, most importantly in the temperatures of denaturation $\left(72 \mathrm{vs} .82^{\circ} \mathrm{C}\right.$, respectively), the hybridization ( 37 vs. $55^{\circ} \mathrm{C}$, respectively) and stringent wash ( $42 \mathrm{vs.} 55^{\circ} \mathrm{C}$, respectively). The lower temperature of ff-GISH might be more appropriate and might prevent DNA loss from small chromosomes, as observed in Melampodium (chromosome size $0.85-2 \mu \mathrm{m}$ in $M$. nayaritense) [Weiss-Schneeweiss et al., 2009], in comparison to medium-sized chromosomes of maize or Tragopogon $(2.5-6 \mu \mathrm{m})$ [Chester et al., 2012] or larger chromosomes of Prospero. Additionally, the protocol optimized for Tragopogon recommends longer hybridization times to increase probe signal intensity $(36-48 \mathrm{~h})$. The current ff-GISH protocol does not require prolonged hybridization times. Hybridization efficiency after $12 \mathrm{~h}$ does not differ significantly from hybridization after $24 \mathrm{~h}$. Both protocols can thus be applied to discriminate closely related sub-genomes, although ff-GISH protocols would most likely perform better for taxa with small chromosomes and genomes.

Both of the labeled genomic parental DNAs used for ff-GISH bind to chromosomes of both parental genomes, albeit with different efficiency. Thus, it is difficult to discriminate the 2 genomes in images from single channels (online suppl. fig. 1). Similarly, the use of only one labeled genomic probe and the use of the other probe as unlabeled blocking DNA did not allow clear discrimination of parental genomes. Only in overlaid images the distinction of the 2 parental genomes is clearly visible (online suppl. fig. 1), similar in its principle to comparative genomic hybridization (CGH) [Pinkel and Albertson, 2005]. The ffGISH signals in overlaid images are the result of differential saturation of 2 colors ( 2 probes labeled with different labels). A higher intensity of one of the probes' signals in a specific region of the given chromosome/whole chromosome indicates the prevalence of this parental genome-specific repeats and usually corresponds to less effective labeling of this region by the other parental genomic DNA.

This differential labeling of chromosomes with both colors indicates that the hybridization does not exclusively rely on preferential mapping of taxon-specific repetitive DNA sequences, similar to standard FISH/GISH. Additionally, particularly in the absence of low levels of unique sequence types, differential mapping of shared/ similar sequence types provides a majority of differentiation. Shared types of repeats of both parental genomes will thus compete for the same/very similar targets, and the final differentiation will result from the efficiency of their competition, provided there is enough saturation and a minimal level of repetitive DNA fraction differentiation $(<99 \%$ of repetitive fraction similarity in Prospero).

\section{Acknowledgments}

The authors thank Dr. Jörg Fuchs (IPK Gatersleben, Germany) and Dr. Harry Scherthan (Bundeswehr Institute of Radiobiology, Germany) for discussions and critical reading of the manuscript. This work was supported by the Austrian Science Fund (projects P21440 and P25131 to H.W.-S.). 


\section{References}

Berndt A, Kosmehl H, Celeda D, Katenkamp D: DEmadzade K, Jang TS, Macas J, Kovařík A, Novák Reduced formamide content and hybridization temperature results in increased non-radioactive mRNA in situ hybridization signals. Acta Histochem 98:79-87 (1996).

Birchler JA, Albert SA, Gao Z: Stability of repeated sequence clusters in hybrids of maize as revealed by FISH. Trop Plant Biol 1:34-39 (2008).

Blake RD, Delcourt SG: Thermodynamic effects of formamide on DNA stability. Nucleic Acids Res 24:2095-2103 (1996).

Blöch C, Weiss-Schneeweiss H, Schneeweiss GM, Barfuss MHJ, Rebernig CA, et al: Molecular phylogenetic analyses of nuclear and plastid DNA sequences support dysploid and polyploidy chromosome number changes and reticulate evolution in the diversification of Melampodium (Millerieae, Asteraceae). Mol Phylogen Evol 53:220-233 (2009).

-Chester M, Gallagher JP, Symonds VV, Cruz da Silva AV, Mavrodiev EV, et al: Extensive chromosomal variation in a recently formed natural allopolyploid species, Tragopogon miscellus (Asteraceae). Proc Natl Acad Sci USA 109:1176-1181 (2012).

Chester M, Riley RK, Soltis PS, Soltis DE: Patterns of chromosomal variation in natural populations of the neoallotetraploid Tragopogon mirus (Asteraceae). Heredity 114:309-317 (2015).

Choi HW, Kim JS, Lee SH, Bang JW: Physical mapping by FISH and GISH of rDNA loci and discrimination of genomes A and B in Scilla scilloides complex distributed in Korea. J Plant Biol 51:408-412 (2008).

Doyle JJ, Doyle JL: A rapid DNA isolation procedure for small quantities of fresh leaf tissue. Phytochem Bull 19:11-15 (1987).

$\mathrm{P}$, et al: Differential amplification of satellite PaB6 in chromosomally hypervariable Prospero autumnale complex (Hyacinthaceae). Ann Bot 114:1597-1608 (2014).

Jang TS, Emadzade K, Parker J, Temsch EM, Leitch AR, et al: Chromosomal diversification and karyotype evolution of diploids in the cytologically diverse genus Prospero (Hyacinthaceae). BMC Evol Biol 13:136 (2013).

Jeridi M, Bakry F, Escoute J, Fondi E, Carreel F, et al: Homoeologous chromosome pairing between the A and B genomes of Musa spp. revealed by genomic in situ hybridization. Ann Bot 108:975-981 (2011).

Kato A, Lamb JC, Birchler JA: Chromosome painting using repetitive DNA sequences as probes for somatic chromosome identification in maize. Proc Natl Acad Sci USA 101: 13554-13559 (2004).

Lihová J, Kučera J, Perný, Marhold K: Hybridization between two polyploid Cardamine (Brassicaceae) species in North-western Spain: discordance between morphological and genetic variation patterns. Ann Bot 99:1083-1096 (2007).

Lim KY, Kovařík A, Matyášek R, Chase MW, Clarkson JJ, et al: Sequence of events leading to near-complete genome turnover in allopolyploid Nicotiana within five million years. New Phytol 175:756-763 (2005).

Mandáková T, Marhold K, Lysak MA: The widespread crucifer species Cardamine flexuosa is an allotetraploid with a conserved subgenomic structure. New Phytol 201:982-992 (2014).

$\checkmark$ Matthiesen SH, Hansen CM: Fast and non-toxic in situ hybridization without blocking of repetitive sequences. PLoS One 7:e40675 (2012).

Meinkoth J, Wahl G: Hybridization of nucleic acids immobilized on solid supports. Anal Bio-

Cremer C: Optimization of fast-fluorescence in situ hybridization with repetitive $\alpha$-satellite probes. Z Naturforsch C 51:253-261 (1996). chem 138:267-284 (1984).

Pendinen G, Gavrilenko T, Jiang J, Spooner DM: Allopolyploid speciation of the Mexican tetraploid potato species Solanum stoloniferum and $S$. hjertingii revealed by genomic in situ hybridization. Genome 51:714-720 (2008).
Pinkel D, Albertson DG: Comparative genomic hybridization. Annu Rev Genomics Hum Genet 6:331-354 (2005).

Renny-Byfield S, Ainouche M, Leitch IJ, Lim KY, Le Comber SC, Leitch AR: Flow cytometry and GISH reveal mixed ploidy populations and Spartina nonaploids with genomes of $S$. alterniflora and S. maritima origin. Ann Bot 105:527-533 (2010).

Schwarzacher T, Heslop-Harrison P: Practical in Situ Hybridization (BIOS Scientific Publishers, Oxford 2000).

Schwarzacher T, Leitch AR, Bennett MD, HeslopHarrison JS: In situ localization of parental genomes in a wide hybrid. Ann Bot 63:315324 (1989).

Soltis PS, Soltis DE: The role of hybridization in plant speciation. Annu Rev Plant Biol 60:561588 (2009).

Stuessy, TF, Blöch C, Villaseñor JL, Rebernig CA, Weiss-Schneeweiss $\mathrm{H}$ : Phylogenetic analyses of DNA sequences with chromosomal and morphological data confirm and refine sectional and series classification within Melampodium (Asteraceae, Millerieae). Taxon 60: 436-449 (2011).

Taylor S: Chromosomal evolution of Scilla autumnalis ( $\mathrm{PhD}$ thesis, University of London 1997)

Vaughan HE, Taylor S, Parker JS: The ten cytological races of the Scilla autumnalis species complex. Heredity 79:371-379 (1997).

Weiss-Schneeweiss H, Villaseñor JL, Stuessy TF: Chromosome numbers, karyotypes, and evolution in Melampodium (Asteraceae). Int J Plant Sci 170:1168-1182 (2009).

Weiss-Schneeweiss H, Blöch C, Turner B, Villaseñor JL, Stuessy TF, Schneeweiss GM: The promiscuous and the chaste: frequent allopolyploid speciation and its genomic consequences in American daisies (Melampodium Sect. Melampodium; Asteraceae). Evolution 66: 211-228 (2012). 